\title{
A Survival Prediction Nomogram for Esophageal Squamous Cell Carcinoma Treated with Neoadjuvant Chemoradiotherapy Followed by Surgery
}

\author{
Tianyan Ding ${ }^{1,2, *}$ \\ Cantong Liu ${ }^{1,2, *}$ \\ Binliang Huang ${ }^{1,2}$ \\ Lingyu Chu (D) ${ }^{1,2}$ \\ Laifeng Wei ${ }^{1,2}$ \\ Yiwei $\operatorname{Lin}^{1,2}$ \\ Yun Luo ${ }^{1,2}$ \\ Biao Zhang ${ }^{1,2}$ \\ Chaoqun Hong ${ }^{3}$ \\ Yiwei Xu (D) ${ }^{1,2}$ \\ Yuhui Peng ${ }^{1,2}$

\begin{abstract}
'Department of Clinical Laboratory Medicine, Cancer Hospital of Shantou University Medical College, Shantou, People's Republic of China; ${ }^{2}$ Precision Medicine Research Center, Shantou University Medical College, Shantou, People's Republic of China; ${ }^{3}$ Guangdong Provincial Key Laboratory of Breast Hospital of Shantou University Medical College, Shantou, People's Republic of China
\end{abstract} \\ Cancer Diagnosis and Treatment, Cancer
}

*These authors contributed equally to this work

Correspondence: Yiwei Xu Department of Clinical Laboratory Medicine, Cancer Hospital of Shantou University Medical College, 7 Raoping Road, Shantou, 5I 504I, Guangdong, People's Republic of China

Fax +86-754-8856-0352

Email yiwei512@126.com

Yuhui Peng

Email pengyuhui666@163.com
Background: Neoadjuvant chemoradiotherapy (NCRT) followed by surgery is a component of the standard treatment for resectable locally advanced esophageal squamous cell carcinoma (ESCC), and the parameters for survival prediction are not clear yet. Our study aimed to construct a survival prediction nomogram for ESCC with NCRT followed by surgery.

Methods: We analyzed hematological parameters and related-derivative indexes from 122 ESCC patients treated with NCRT followed by surgery. Univariate and multivariate Cox survival analyses were performed to identify independent prognostic factors to establish a nomogram and predict overall survival (OS). The predictive value of the nomogram for OS was evaluated by the concordance index (C-index), decision curve analysis (DCA), the clinical impact curve (CIC), net reclassification improvement (NRI), and integrated discrimination improvement (IDI).

Results: The pretreatment nutritional candidate, prognostic nutrition index, inflammationrelated absolute monocyte count and TNM staging were entered into the nomogram for ESCC with NCRT followed by surgery. The C-index of the nomogram for OS was $0.790(95 \% \mathrm{CI}=$ $0.688-0.893)$, which was higher than that of TNM staging $(0.681 ; 95 \% \mathrm{CI}=0.565-0.798, \mathrm{P}=$ 0.026). The DCA, CIC, NRI, and IDI of the nomogram showed moderate improvement in predicting survival. Based on the cut point calculated according to the constructed nomogram, the high-risk group had poorer OS than that of the low-risk group $(\mathrm{P}<0.05)$.

Conclusion: A novel nomogram based on nutrition- and inflammation-related indicators might help predict the survival of ESCC treated with NCRT followed by surgery.

Keywords: esophageal squamous cell carcinoma, neoadjuvant chemoradiotherapy, surgery, nomogram, survival, prognosis

\section{Introduction}

Esophageal cancer (EC) is one of the most common malignant tumors in the world. The prognosis of EC remains poor with the overall 5-year survival rates ranging from $15 \%$ to $25 \%{ }^{1,2}$ Esophageal squamous cell carcinoma (ESCC) is the most frequent histological subtype, with the highest incidence in some parts of Asia. China alone accounts for more than half of global cases. ${ }^{3}$

Currently, the optimal treatment for ESCC is a debatable point. Surgery is still recommended as the preferred curative treatment. For locally advanced ESCC, neoadjuvant chemoradiotherapy (NCRT) followed by surgery has been proven to achieve more favorable long-term survival than surgery alone. ${ }^{4-6}$ Strong evidence suggests that NCRT is expected to kill the micro-metastasis and demote staging to make the operation easier and improve the radical resection rate. ${ }^{7,8}$ Furthermore, NCRT offers a potential opportunity for evaluation of tumor sensitivity to chemotherapy drugs in vivo. However, there 
are still some risks in NCRT. This approach is associated with toxicity, which can contribute to subsequent post-operative morbidity and mortality. ${ }^{9-12}$ It will also miss the critical opportunity of surgical resection for ESCC patients who are ineffective in NCRT. The Union for International Cancer Control tumor/node/metastasis (TNM) staging system is a widely used tool for predicting the outcome of ESCC. ${ }^{13}$ However, the TNM staging is sometimes not accurate because it may happen that some patients with similar TNM stages exhibit inconsistent clinical survival outcomes. ${ }^{14-18}$ Therefore, it is necessary to establish a prediction model with additional prognostic factors for these patients treated with NCRT followed by surgery for further study.

In recent years, host immune and inflammatory responses have been considered as a marker of cancer progression and prognosis, ${ }^{19-22}$ which can be evaluated by hematological parameters, such as monocyte-to-lymphocyte ratio (MLR) and the absolute monocyte count (AMC). Monocytes play an important role in the inflammatory response produced by tumors. Tumor-associated macrophages (TAMs) derived from circulating monocytes are recruited to the tumor sites by chemotactic factors. TAMs could release many effective angiogenic and lymphangiogenic growth factors, cytokines to enhance angiogenesis and lymphangenesis and promote the invasion and metastasis of cancer cells. ${ }^{23}$ Epidemiological study revealed that elevated circulating TAMs are associated with poor prognosis in ESCC. ${ }^{24}$

Moreover, prognostic nutrition index (PNI) is a nutritional assessment and risk prediction established by Japanese scholar Onodera. ${ }^{25}$ At present, it is mainly used to evaluate the malignant degree and prognosis of digestive tract tumors such as esophageal cancer, ${ }^{26,27}$ gastric cancer $^{28}$ and pancreatic cancer. ${ }^{29,30}$ Previous studies have showed that malnutrition is associated with an immunosuppressed condition, which provides a good microenvironment for tumor recurrence. ${ }^{31,32}$ Nakatani et al reported that PNI is associated with tumor progression and survival in patients with esophageal cancer. ${ }^{33}$ However, to our knowledge, there is no study of PNI on the evaluation of ESCC with NCRT followed by surgery. ${ }^{34}$ Systemic immune-inflammation index (SII), a new blood-derived inflammatory index, is also considered to be associated with poor outcome in ESCC. ${ }^{35}$

In view of the prospect of hematological immunoinflammation and nutrition biomarkers in predicting survival, we established a novel nomogram for ESCC treated with NCRT followed by surgery and assessed its incremental value to the traditional staging system and clinical treatment for OS.

\section{Methods and Materials}

\section{Study Population}

We retrospectively analyzed a total of 122 patients with ESCC treated with NCRT followed by surgery, who were recruited from the Cancer Hospital of Shantou University Medical College from 2007 to 2021 . We reviewed the detailed medical records of these patients who were diagnosed based on spiral computed-tomography (CT) and endoscopic examination followed by histopathology. According to the results of the medical examinations, patients were tumor-node-metastasis (TNM) staged based on the 8th International Union Against Cancer (UICC) criteria for esophageal carcinoma. ${ }^{36}$

Patients included in the analysis met the following criteria: (1) they were diagnosed as ESCC with histopathological examination; (2) they did not suffer from previous or concomitant malignancies before ESCC diagnosis or receive any anti-cancer treatment; (3) they underwent chest CT examination, and the lung metastatic lesions could be ruled out; (4) they had received NCRT before esophageal cancer surgery; (5) they had complete baseline clinical information, laboratory, and follow-up data. We excluded those patients who died of surgical complications. The OS was defined as the interval between the initial diagnosis and either death of cancer or the last follow-up. In this study, all blood test results were obtained before treatment. This study was approved by the Hospital Ethics Committee in Shantou University Cancer Center. The requirement for informed consent from patients was waived because of its retrospective design. All work was complied with the principles of the Helsinki Declaration.

\section{Neoadjuvant Chemoradiotherapy and Surgery}

Neoadjuvant therapy comprised weekly carboplatin (AUC $2 \mathrm{mg} / \mathrm{mL}$ per $\mathrm{min})$ and paclitaxel $\left(50 \mathrm{mg} / \mathrm{m}^{2}\right)$ for 5 weeks combined with daily radiotherapy consisting of 23 fractions of $1.8 \mathrm{~Gy}$ (total $41.4 \mathrm{~Gy}$ ). All patients having treatment with curative intent received neoadjuvant chemoradiotherapy unless considered unfit for multimodal treatment. ${ }^{5}$ For patients with tumors mainly involving the gastroesophageal junction, transabdominal resection is beneficial. ${ }^{37}$ For intrathoracic esophageal tumors and connective tumors with positive cervical lymph nodes or above, transthoracic lymph node dissection is usually performed. All operations were performed or strictly supervised by experienced upper gastrointestinal surgeons. 


\section{Parameters for the Establishment of \\ Nomogram}

The following relevant clinicopathological and hematological data were collected for each enrolled patient at the time of diagnosis and before any treatment: gender, age, body mass index (BMI), tumor location, TNM stage, prognostic nutrition index (PNI), systemic immune-inflammation index (SII), white blood cell (WBC), red blood cell (RBC), platelet count (PLT), Hemoglobin (HB), absolute monocyte count (AMC), absolute neutrophil count (ANC), absolute lymphocyte count (ALC), platelet lymphocyte ratio (PLR), neutrophil lymphocyte ratio (NLR) and lymphocyte monocyte ratio (LMR). The clinical stage of the disease was determined according to 8th edition of the AJCC TNM stage manual. ${ }^{38}$ PNI was calculated by the formula Alb $(\mathrm{g} / \mathrm{L})+5 \times$ lymphocyte count $\left(\times 10^{9} / \mathrm{L}\right)$. SII was calculated by the formula PLT $\left(\times 10^{9} / \mathrm{L}\right) \times$ Neutrophil Count $\left(\times 10^{9} / \mathrm{L}\right) \div$ lymphocyte count $\left(\times 10^{9} / \mathrm{L}\right)$. In this study, continuous variables were transformed into categorical variables. We used a graphical method, the X-tile plot that shows the robustness of the relationship between a biomarker and outcome by construction of a twodimensional projection of every possible subpopulation. The best cut-off values for all variables were determined by X-tile. ${ }^{39}$

\section{Statistical Analyses}

Statistical analyses were performed using SPSS software, version 19.0 (IBM Corp., Chicago, IL, USA) and R (version 4.0.3, http://www.R-project.org) for Windows. The KaplanMeier curves were used to calculate the survival rate, and the Log rank test was used to compare them. Univariate analysis was to select the most useful prognostic variables. Variables with a significant level of $\mathrm{P} \leq 0.1$ in univariate analysis were analyzed using multivariate Cox regression. A dynamic predictive nomogram model is built using all variables with a P-value of less than 0.05 in a multivariate model. The discriminative ability, accuracy and incremental predictive value of the prognostic nomogram to the traditional TNM staging system for individualized survival was evaluated by the Harrell's concordance index (C-index), decision curve analysis (DCA) ${ }^{40}$ net reclassification improvement (NRI), ${ }^{41}$ and integrated discrimination improvement (IDI). ${ }^{42}$ Throughout the study, statistical significance was set at $\mathrm{P}<$ 0.05 (two-tailed).

\section{Results}

\section{Patient Characteristics}

In our study, a total of 122 eligible patients were analyzed. The median follow-up was 22.0 months (interquartile range (IQR): $12.0-42.0$ ). The median age for these patients was 59 years (IQR: 55-64 years), of which 98 (80.3\%) were males

Table I Patient Demographics and Clinical Characteristics

\begin{tabular}{|c|c|c|c|c|c|}
\hline Characteristics & No & $\%$ & Characteristics & No & $\%$ \\
\hline Gender & & & $\operatorname{PLT}\left(10^{9} / \mathrm{L}\right)$ & & \\
\hline Male & 98 & 80.3 & $\leq 338$ & 95 & 77.9 \\
\hline Female & 24 & 19.7 & $>338$ & 27 & 22.1 \\
\hline Age (years) & & & $\operatorname{AMC}\left(10^{9} / \mathrm{L}\right)$ & & \\
\hline$\leq 62$ & 85 & 69.7 & $\leq 0.6$ & 70 & 57.4 \\
\hline$>62$ & 37 & 30.3 & $>0.6$ & 52 & 42.6 \\
\hline BMI & & & $\operatorname{ANC}\left(10^{9} / \mathrm{L}\right)$ & & \\
\hline$\leq 21.2$ & 67 & 54.9 & $\leq 5.0$ & 68 & 55.7 \\
\hline$>21.2$ & 55 & 45.1 & $>5.0$ & 54 & 44.3 \\
\hline Location & & & $\operatorname{ALC}\left(10^{9} / \mathrm{L}\right)$ & & \\
\hline Up & 27 & 22.1 & $\leq 1.7$ & 51 & 41.8 \\
\hline Middle & 81 & 66.4 & $>1.7$ & 71 & 58.2 \\
\hline Low & 14 & 11.5 & PLR & & \\
\hline TNM stage & & & $\leq 153.3$ & 58 & 47.5 \\
\hline II & 10 & 8.2 & $>153.3$ & 64 & 52.5 \\
\hline III & 57 & 46.7 & NLR & & \\
\hline $\mathrm{IVa}$ & 49 & 40.2 & $\leq 2.3$ & 47 & 38.5 \\
\hline $\mathrm{IVb}$ & 6 & 4.9 & $>2.3$ & 75 & 61.5 \\
\hline PNI & & & LMR & & \\
\hline$\leq 50.5$ & 106 & 86.9 & $\leq 3.4$ & 59 & 48.4 \\
\hline$>50.5$ & 16 & 13.1 & $>3.4$ & 63 & 51.6 \\
\hline \multicolumn{6}{|l|}{ SII } \\
\hline$\leq 852.9$ & 69 & 56.6 & & & \\
\hline$>852.9$ & 53 & 43.4 & & & \\
\hline \multicolumn{6}{|l|}{ WBC $\left(10^{9} / \mathrm{L}\right)$} \\
\hline$\leq 7.6$ & 64 & 52.5 & & & \\
\hline$>7.6$ & 58 & 47.5 & & & \\
\hline \multicolumn{6}{|l|}{$\operatorname{RBC}\left(10^{12} / L\right)$} \\
\hline$\leq 4.5$ & 42 & 34.4 & & & \\
\hline$>4.5$ & 80 & 65.6 & & & \\
\hline \multicolumn{6}{|l|}{$\mathrm{HB}(g / L)$} \\
\hline$\leq 132.7$ & 52 & 42.6 & & & \\
\hline$>132.7$ & 70 & 57.4 & & & \\
\hline
\end{tabular}

Abbreviations: BMI, body mass index; PNI, prognostic nutrition index; SII, systemic immune-inflammation index; WBC, white blood cell; RBC, red blood cell; HB, hemoglobin; PLT, platelet count; AMC, absolute monocyte count; ANC, absolute neutrophil count; ALC, absolute lymphocyte count; PLR, platelet lymphocyte ratio; NLR, neutrophil lymphocyte ratio; LMR, lymphocyte monocyte ratio. 
and $24(19.7 \%)$ were females. There were 27, 81 and 14 patients with esophageal neoplasm located in upper, middle and lower chest, respectively. Patient demographic and clinical characteristics are summarized in Table 1 . The best cutoff values for all variables were as follows: age (62 years), BMI (21.2), PNI (50.5), SII (852.9), WBC $\left(7.6 \times 10^{9} / \mathrm{L}\right)$, RBC $\left(4.5 \times 10^{12} / \mathrm{L}\right), \quad$ PLT $\left(338 \times 10^{9} / \mathrm{L}\right), \quad \mathrm{HB}(132.7 \mathrm{~g} / \mathrm{L})$, AMC $\left(0.6 \times 10^{9} / \mathrm{L}\right)$, ANC $\left(5.0 \times 10^{9} / \mathrm{L}\right)$, ALC $\left(1.7 \times 10^{9} / \mathrm{L}\right)$, PLR (153.3), NLR (2.3) and LMR (3.4).

\section{Cox Proportional Hazards Regression Analysis of the Overall Survival}

As shown in Table 2, the univariate analysis indicates that TNM stage $(\mathrm{P}=0.012)$, PNI $(\mathrm{P}=0.010)$, SII $(\mathrm{P}=0.088)$, WBC $(\mathrm{P}=0.044), \operatorname{PLT}(\mathrm{P}=0.028), \mathrm{AMC}(\mathrm{P}=0.019), \mathrm{ANC}$
$(\mathrm{P}=0.040)$ and HB $(\mathrm{P}=0.083)$ were associated with OS of patients. Then they were included in the multivariate Cox proportional risk regression analysis of OS. The results show that the following variables remained independently prognostic: PNI ( $\mathrm{P}=0.006, \mathrm{HR}=3.986$; 95\% CI: 1.488-10.677), AMC $(\mathrm{P}=0.047, \mathrm{HR}=2.569 ; 95 \% \mathrm{CI}: 1.013-6.516)$ and TNM stage $(\mathrm{P}=0.008, \mathrm{HR}=2.618 ; 95 \% \mathrm{CI}: 1.280-5.313)$. According to Cox proportional hazards regression analysis, the forest plot shows the hazard ratios and $95 \%$ confidence intervals for OS (Figure 1).

\section{Construction of the Multi-Parametric Prognostic Nomogram}

Using these selected markers, the nomogram was constructed for OS prediction (Figure 2). From the nomogram,

Table 2 Univariate and Multivariate Cox Proportional Hazards Regression Analysis for OS

\begin{tabular}{|c|c|c|c|c|}
\hline \multirow[t]{2}{*}{ Characteristics } & \multicolumn{2}{|c|}{ Univariate Analysis } & \multicolumn{2}{|c|}{ Multivariate Analysis } \\
\hline & HR (95\% CI) & $P$ value & HR (95\% Cl) & $P$ value \\
\hline \multicolumn{5}{|l|}{ Gender } \\
\hline Male & $0.512(0.148-1.775)$ & 0.291 & & \\
\hline Female & Reference & & & \\
\hline \multicolumn{5}{|l|}{ Age (years) } \\
\hline$\leq 62$ & $0.698(0.252-1.930)$ & 0.487 & & \\
\hline$>62$ & Reference & & & \\
\hline \multicolumn{5}{|l|}{ BMI } \\
\hline$\leq 21.2$ & $0.496(0.187-1.317)$ & 0.159 & & \\
\hline$>21.2$ & Reference & & & \\
\hline \multicolumn{5}{|l|}{ Location } \\
\hline Up & $1.584(0.319-7.874)$ & 0.574 & & \\
\hline Middle & I.I54 (0.257-5.184) & 0.853 & & \\
\hline Low & Reference & & & \\
\hline TNM stage & & & $2.608(1.280-5.3 \mid 3)$ & 0.008 \\
\hline II & $0.067(0.007-0.663)$ & 0.021 & & \\
\hline III & $0.089(0.02 \mathrm{I}-0.382)$ & 0.001 & & \\
\hline $\mathrm{IVa}$ & $0.173(0.046-0.654)$ & 0.010 & & \\
\hline $\mathrm{IVb}$ & Reference & & & \\
\hline $\mathrm{PNI}$ & & & $3.986(1.488-10.677)$ & 0.006 \\
\hline$\leq 50.5$ & $3.804(1.443-10.02)$ & 0.010 & & \\
\hline$>50.5$ & Reference & & & \\
\hline \multicolumn{5}{|l|}{ SII } \\
\hline$\leq 852.9$ & $2.190(0.890-5.389)$ & 0.088 & & \\
\hline$>852.9$ & Reference & & & \\
\hline \multicolumn{5}{|l|}{ WBC $\left(10^{9} / \mathrm{L}\right)$} \\
\hline$\leq 7.6$ & $2.753(1.028-7.375)$ & 0.044 & & \\
\hline$>7.6$ & Reference & & & \\
\hline
\end{tabular}

(Continued) 
Table 2 (Continued).

\begin{tabular}{|c|c|c|c|c|}
\hline \multirow[t]{2}{*}{ Characteristics } & \multicolumn{2}{|c|}{ Univariate Analysis } & \multicolumn{2}{|c|}{ Multivariate Analysis } \\
\hline & HR (95\% Cl) & $P$ value & HR (95\% Cl) & $P$ value \\
\hline \multicolumn{5}{|l|}{$\operatorname{RBC}\left(10^{12} / \mathrm{L}\right)$} \\
\hline$\leq 4.5$ & $1.514(0.563-4.07 I)$ & 0.411 & & \\
\hline$>4.5$ & Reference & & & \\
\hline \multicolumn{5}{|l|}{$\mathrm{HB}(\mathrm{g} / \mathrm{L})$} \\
\hline$\leq 132.7$ & $0.443(0.176-1.112)$ & 0.083 & & \\
\hline$>132.7$ & Reference & & & \\
\hline \multicolumn{5}{|l|}{ PLT (109\%/L) } \\
\hline$\leq 338$ & $2.793(1.121-6.961)$ & 0.028 & & \\
\hline$>338$ & Reference & & & \\
\hline $\operatorname{AMC}\left(10^{9} / \mathrm{L}\right)$ & & & $2.569(1.013-6.516)$ & 0.047 \\
\hline$\leq 0.6$ & $3.026(1.202-7.618)$ & 0.019 & & \\
\hline$>0.6$ & Reference & & & \\
\hline \multicolumn{5}{|l|}{ ANC $\left(10^{9} / \mathrm{L}\right)$} \\
\hline$\leq 5.0$ & $2.807(1.048-7.514)$ & 0.040 & & \\
\hline$>5.0$ & Reference & & & \\
\hline \multicolumn{5}{|l|}{$\operatorname{ALC}\left(10^{9} / \mathrm{L}\right)$} \\
\hline$\leq 1.7$ & $2.07(0.785-5.454)$ & 0.141 & & \\
\hline$>1.7$ & Reference & & & \\
\hline \multicolumn{5}{|l|}{ PLR } \\
\hline$\leq 153.3$ & $1.375(0.562-3.367)$ & 0.485 & & \\
\hline$>153.3$ & Reference & & & \\
\hline \multicolumn{5}{|l|}{ NLR } \\
\hline$\leq 2.3$ & $\mathrm{I} .63(0.624-4.26 \mathrm{I})$ & 0.319 & & \\
\hline$>2.3$ & Reference & & & \\
\hline \multicolumn{5}{|l|}{ LMR } \\
\hline$\leq 3.4$ & $0.532(0.21 \mid-1.342)$ & 0.181 & & \\
\hline$>3.4$ & Reference & & & \\
\hline
\end{tabular}

Abbreviations: OS, overall survival; BMI, body mass index; PNI, prognostic nutrition index; SIl, systemic immune-inflammation index; WBC, white blood cell; RBC, red blood cell; HB, hemoglobin; PLT, platelet count; AMC, absolute monocyte count; ANC, absolute neutrophil count; ALC, absolute lymphocyte count; PLR, platelet lymphocyte ratio; NLR, neutrophil lymphocyte ratio; LMR, lymphocyte monocyte ratio.

TNM stage had the greatest impact on OS, followed by PNI and AMC. A larger total point score indicates a shorter OS. The nomogram was used by summing the points identified on the points scale for each variable. The total points projected on the bottom scales indicate the probability of 1-, 3-, and 5-year survival. For example, a patient, regardless of age or sex, with PNI $>56.4$, AMC $>0.6 \times 10^{9} / \mathrm{L}$, and TNM stage II had a total of 75 points indicating an estimated 1-, 3-, and 5-year OS of $87 \%$, $70 \%$, and $66 \%$, respectively. The calibration plots for the probability of survival at 1-, 3-, and 5-year showed the prediction of the nomogram was well matched with the actual observation (Figure S1A- ).

\section{Assessment of Performance of Prognostic Nomogram}

Nomogram discrimination was evaluated using the C-index, which enumerated the level of concordance between the predicted and observed OS. From Table 3, the C-index based on the nomogram $(0.790 ; 95 \% \mathrm{CI}$, 0.688-0.893) for OS in the cohort was much higher than that of the TNM stage $(0.681 ; 95 \% \mathrm{CI}, 0.565-0.798 ; \mathrm{P}=$ 0.026). The nomogram showed better discrimination than a single component to predict OS (Figure 3).

Due to the small sample size in this study, we applied DCA to evaluate the net benefit of the nomogram and NRI and IDI to assess predictive accuracy. The DCA curve suggested that 


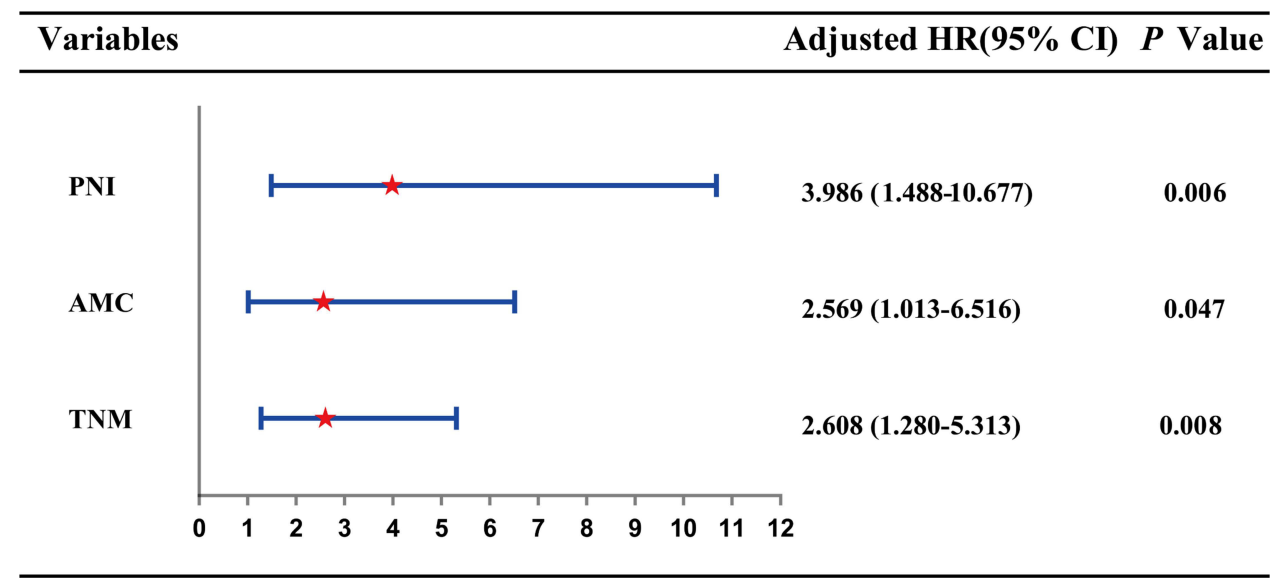

Figure I Forest plot showed the hazard ratio for overall survival according to the Cox proportional hazards regression analysis in ESCC patients. Abbreviation: ESCC, esophageal squamous cell carcinoma.

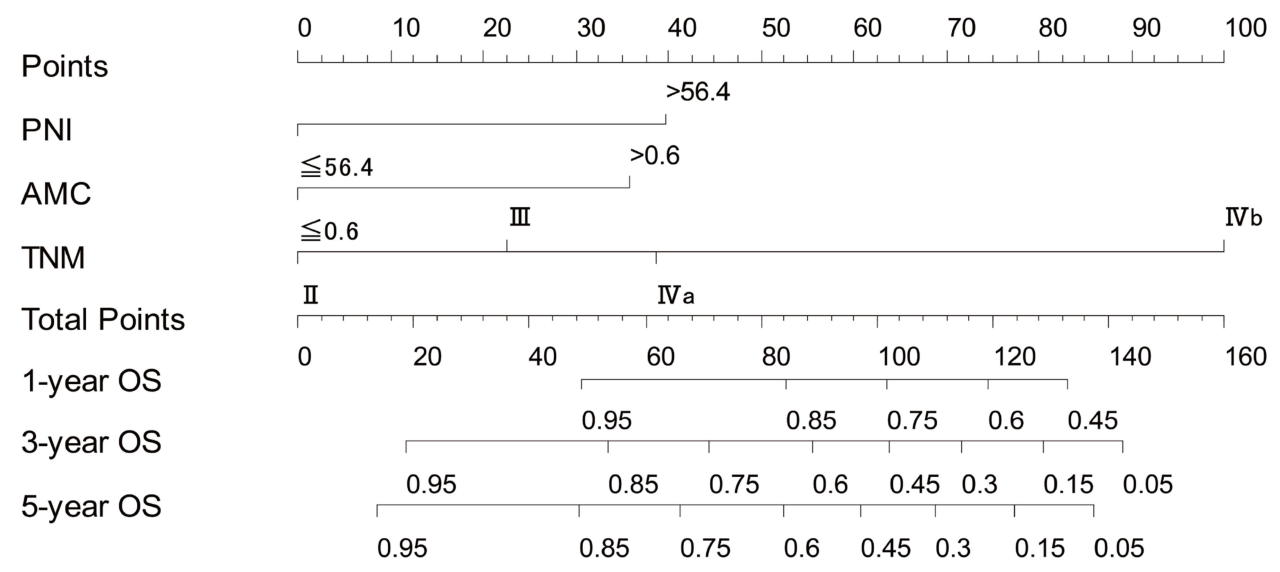

Figure 2 Nomogram model based on PNI, AMC and TNM stage in the prediction of I-, 3- and 5-year overall survival in ESSC patients.

Abbreviations: PNI, prognostic nutrition index; AMC, absolute monocyte count; TNM, tumor/node/metastasis; ESCC, esophageal squamous cell carcinoma.

the nomogram (red line) had a higher net benefit than that of TNM staging (green line) to predict OS (Figure S2). The clinical impact curve also indicated that the nomogram had good net benefits for the identification of severe ESCC patients (Figure S3). In Table 4, the IDI suggested that the predictive

Table 3 C-Index for the Prediction of OS

\begin{tabular}{|l|c|c|}
\hline Factor & C-Index $(95 \%$ Cl) & P value \\
\hline PNI & $0.613(0.509-0.716)$ & \\
AMC & $0.618(0.499-0.737)$ & \\
TNM stage & $0.681(0.565-0.798)$ & \\
Nomogram & $0.790(0.688-0.893)$ & \\
Nomogram vs PNI & & 0.002 \\
Nomogram vs AMC & & $<0.001$ \\
Nomogram vs TNM & & 0.026 \\
\hline
\end{tabular}

Abbreviations: OS, overall survival; PNI, prognostic nutrition index; AMC, absolute monocyte count; TNM, tumor node metastasis. accuracy of the nomogram was better than those of other evaluation systems, including TNM staging (IDI $>0$ ). Furthermore, the NRI showed the accuracy of the nomogram had improvements of $17.6 \%, 38.8 \%$, and $34.9 \%$ when predicting 1-, 3-, and 5-y OS, respectively. To conclude, the DCA, NRI, and IDI indicated a better net benefit and predictive accuracy of the newly constructed model.

\section{Construction of Risk Stratification Based on Nomogram}

Based on cutoff value (67 for OS) of the total points determined by the X-tile program, we subdivided patients into low- and high-risk groups, and applied a KaplanMeier survival analysis to assess their survival. In our cohort, compared with patients in the low-risk group, patients in the high-risk one had shorter OS $(\mathrm{P}<0.01$; Figure 4). This stratification demonstrated that the newly 


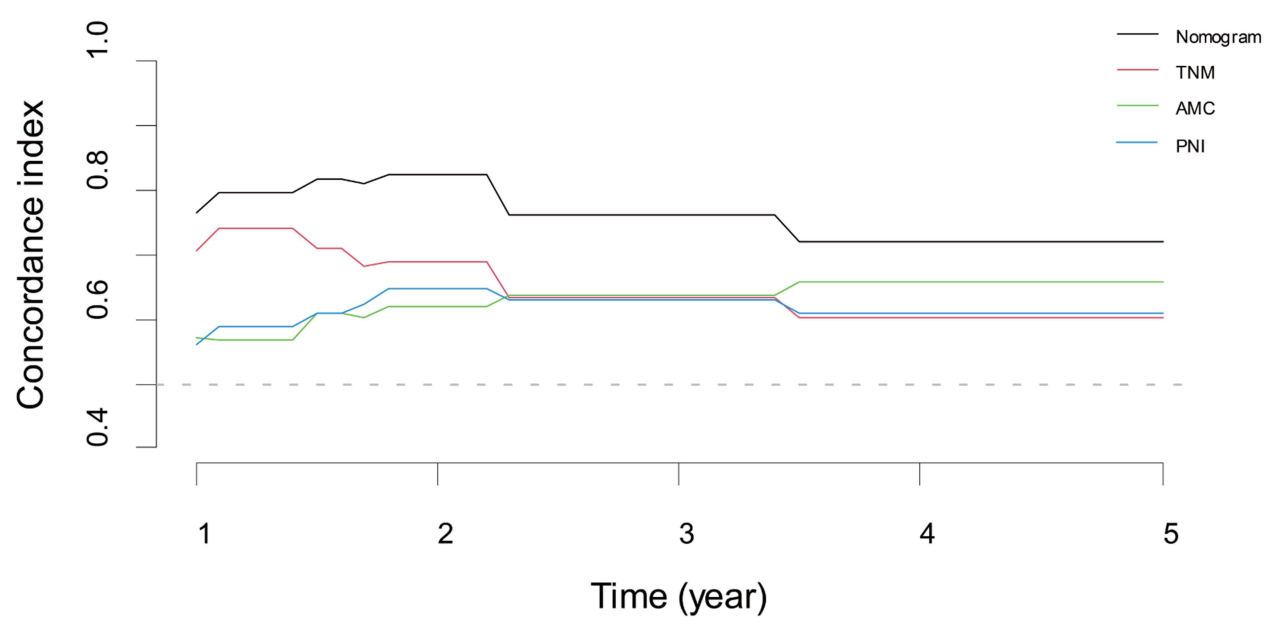

Figure 3 Harrell's concordance index based on the predictions of the nomogram.

constructed nomogram could effectively separate OS for the two proposed risk groups.

\section{Discussion}

NCRT before surgery has been a focus of research for ESCC treatment in recent years. Owing to the high malignancy potential, patients who only receive surgery trend to relapse. NCRT can increase the rate of radical resections and reduce locoregional recurrences. ${ }^{43-45}$ Multidisciplinary comprehensive treatment can prolong the survival time of patients, but the prognosis is still poor.

In the present study, we analyzed individual clinical features and hematological markers and successfully established a prognostic nomogram to predict OS for ESCC treated with NCRT followed by surgery with the use of univariate analysis and multivariate cox proportional hazards regression. The effects of inflammation and nutrition on the prognosis of ESCC patients with NCRT were analyzed, which provided reference for the treatment of esophageal cancer. This nomogram shows better predictive accuracy and discriminative ability in the prognosis of ESCC patients treated with NCRT followed by surgery when compared to traditional TNM staging. Our prognostic nomogram efficiently stratified those patients into high-risk and low-risk subgroups with significant differences in OS.

With the continuous improvement of diagnosis and treatment methods, many malignant tumors have gradually become a controllable chronic disease. Malnutrition in patients with upper gastrointestinal malignancies is frequent due to increased metabolic demand and loss of nutrition. ${ }^{46}$ Meanwhile, the increase of inflammatory cytokines associated with cancer can also lead to malnutrition in cancer patients. The nutrients available in tumor microenvironment plays a dominant role in defining cancer cell metabolism. ${ }^{47,48}$ In fact, although tumor metabolism is highly heterogeneous, some tumors have been proved to develop metabolic dependence on glutamine and other nutrients. Availability of nutrients depends on the flow of plasma nutrients from systemic circulation to tumor cells. ${ }^{49}$ PNI, calculated using serum albumin levels and total lymphocyte count in peripheral blood, was established by Japanese scholar and originally used to evaluate the nutritional and immune status of patients undergoing gastrointestinal surgery. ${ }^{50}$ In recent years, it has gradually become a new index to judge the prognosis of malignant tumors. ${ }^{51-53}$ Many recent studies reveal that there is an association between PNI and survival in various cancers, including colorectal cancer, ${ }^{54}$ gastric cancer, ${ }^{55}$ lung cancer, ${ }^{56-59}$ etc. Uniformly, this study shows that the PNI level is the independent influencing factor of patients' OS. Therefore, nutritional evaluation should be carried out before salvage in patients with NCRT, and the nutritional status of patients with nutritional risk or malnutrition should be improved actively in order to improve long-term survival.

Currently, it has been well established that inflammation has a strong link with cancer development through proliferative responses, invasion, and metastasis. ${ }^{60}$ Tumour cells produce various cytokines that attract leukocytes. The inflammatory component of developing tumors may include different leukocyte populations, such as neutrophils, dendritic cells, lymphocytes, etc. In the presence of granulocyte-macrophage colonization stimulating factor (GM-CSF) and interleukin (IL)-4, Monocytes differentiate into immature dendritic cells. ${ }^{61}$ Dendritic cells migrate to 


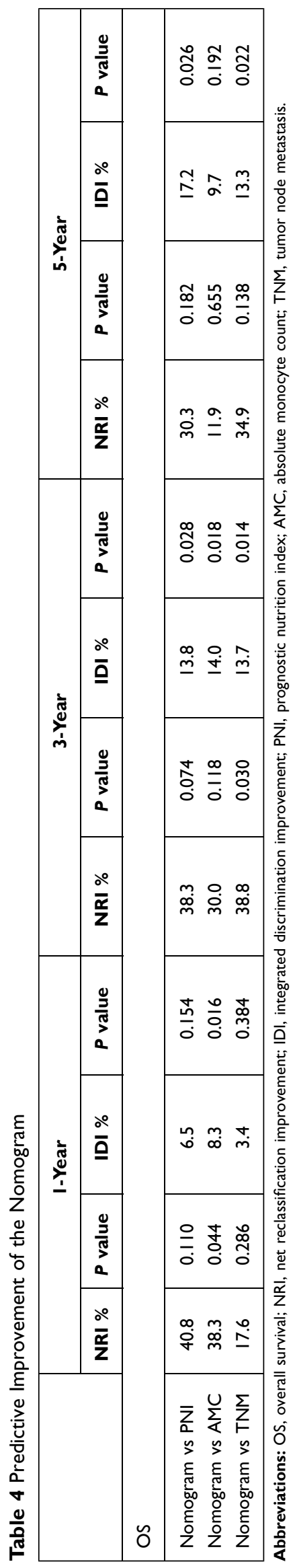

inflammatory peripheral tissues to capture antigens and migrate to lymph nodes to activate $\mathrm{T}$ lymphocytes after maturation. Monocytes seem to be recruited during the whole process of tumor progression, including the early stages of tumor growth and the establishment of distant metastasis. ${ }^{62-64}$ Many studies suggest that a high AMC was associated with tumor poor OS, ${ }^{65-68}$ which was also reflected in our study. Here we show that 1-, 3- and 5-years survival and median survival time in high AMC groups were $87.0 \%, 21.1 \%, 5.7 \%$ and 19 months, respectively. But for the low AMC group, 1-, 3- and 5-years survival and median survival time were $80.0 \%, 35.7 \%, 11.4 \%$ and 23 months, respectively. There is a significant statistical difference between the two groups $(p=0.019)$. Similarly, Han et $\mathrm{al}^{69}$ also found that compared with the preoperative low AMC group, patients with high AMC had poor DFS (high and low: $27.5 \%$ vs $39.0 \%, \mathrm{P}=0.015$ ) and $\mathrm{OS}$ (high and low: $31.1 \%$ vs $44.8 \%, \mathrm{P}=0.009$ ); Preoperative AMC were independent prognostic factors of ESCC for DFS (P $=0.025)$ and $\mathrm{OS}(\mathrm{p}=0.015)$, respectively. These findings may reflect that AMC could be regarded as a substitute biomarker of systemic inflammation, which contributes to tumor progression.

Besides, although some pathological factors such as pathologically complete response (pCR), marginal status and lymph node status are not involved in our modeling process, their influence on the prognosis of esophageal cancer still deserves our great attention. The study indicates approximately $30 \%$ of patients receiving NCRT followed by surgery have a pCR. ${ }^{4}$ It has also been found that early tumors show pCR more often than later tumors after NCRT, which may be due to lower tumor burden. ${ }^{70}$ Compared to patients with residual disease in the resection specimen, patients with pCR have a better overall survival. ${ }^{71,72}$ The resection margin is considered as an important factor in the surgical treatment of esophageal cancer. ${ }^{73}$ Dexter et al studied 135 patients who received esophagogastrectomy and reported that presence of tumor within $1 \mathrm{~mm}$ of the circumferential margin was a significant and independent predictor of survival. This was found to be more significant in patients with a low nodal metastatic burden. $^{73}$ Some retrospective reports imply that lymph node status is also an essential prognostic factor regardless of whether patients received NCRT. ${ }^{74-76}$ However, there is still controversy because of the lack of prospective evidence and the numbers of patients in these studies. 


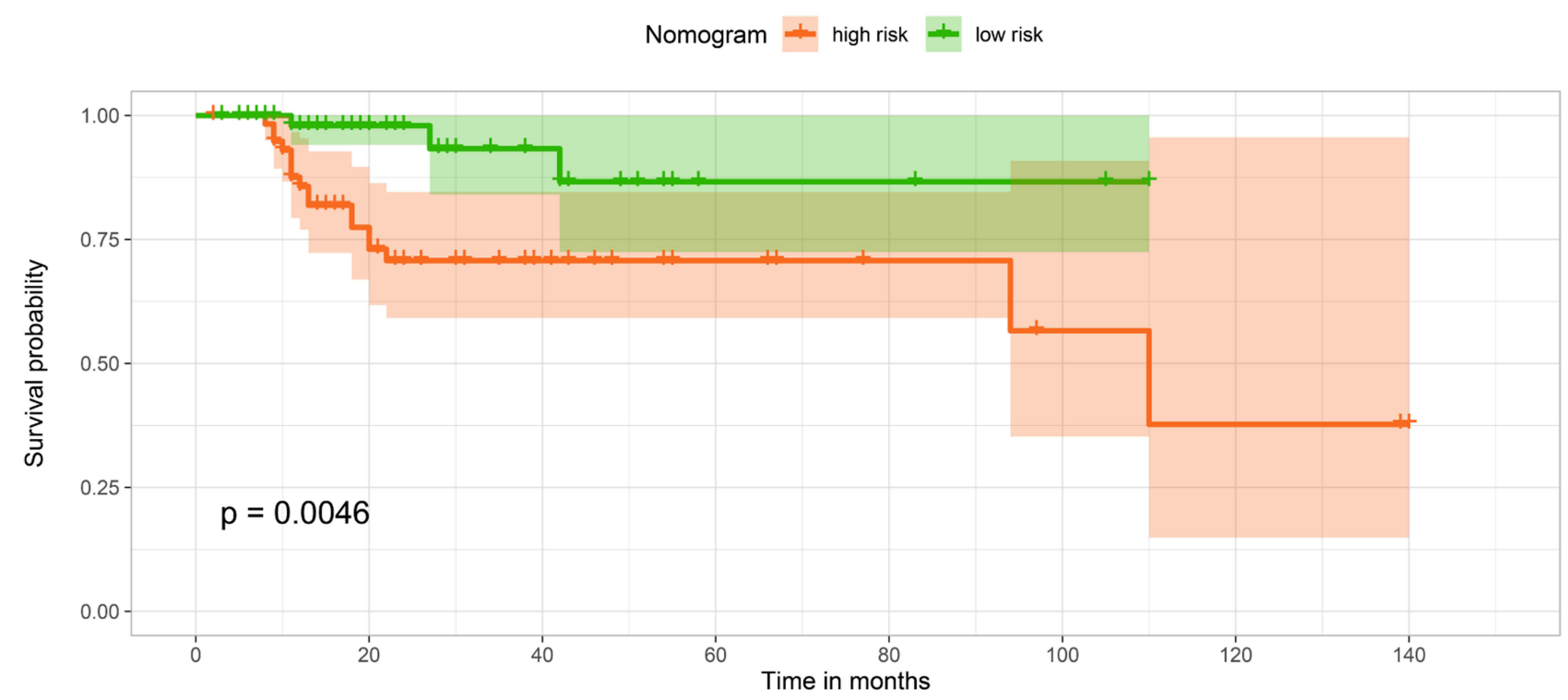

\begin{tabular}{|c|c|c|c|c|c|c|c|c|}
\hline \multirow{4}{*}{ 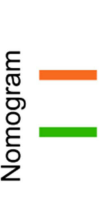 } & \multicolumn{8}{|c|}{ Number at risk: $\mathrm{n}(\%)$} \\
\hline & $64(100)$ & $35(55)$ & $19(30)$ & $8(12)$ & $5(8)$ & $3(5)$ & $2(3)$ & $1(2)$ \\
\hline & $58(100)$ & $31(53)$ & $14(24)$ & $3(5)$ & $3(5)$ & $2(3)$ & $0(0)$ & $0(0)$ \\
\hline & 0 & 20 & 40 & 60 & $\begin{array}{c}80 \\
\text { months }\end{array}$ & 100 & 120 & 140 \\
\hline
\end{tabular}

Figure 4 Kaplan-Meier curves for overall survival based on the predictions of the nomogram.

This study has the following advantages: (1) Compared to previous studies, ${ }^{77-79}$ multiple potential prognostic factors were assessed, thus to increase prognostic accuracy and the C-index of the prognostic nomogram. (2) Compared with the traditional TNM staging system, the new nomogram improved the prognostic prediction ability and accuracy. Although this nomogram might represent a helpful survival prediction tool to assist in therapy decisions, some limitations should not be ignored: (1) At present, we only analyzed small size sample from a single cancer center. A large-scale multi-center verification of results will be needed in the future. (2) For further research, continuous variables need to be transformed into categorical variables based on the cut-off values. There were some limitations in choosing the cut-off values for continuous variables. (3) Our endpoint was OS, and further research on the disease-free survival (DFS) should also be conducted. (4) There may be some changes in the selection of patients for these treatments at this hospital over the study period. We should consider adjusting for year of treatment as a variable in our multivariate model to account for these potential changes. Despite the above-mentioned shortcomings, the prognostic nomogram is effective and may be useful in predicting the outcomes of ESCC treated with NCRT followed by surgery.

\section{Conclusion}

In our study, we established and provided a multiparametric prognostic nomogram derived from nutritionand inflammation-related indicators that showed favorable performance when compared to traditional TNM staging for individualized OS estimation. If further validation in multi-center, large-scale trial studies could be completed, this simple, precise and understandable prognostic model may serve as a potential tool for clinicians in the prognostic prediction of ESCC treated with NCRT followed by surgery.

\section{Abbreviations}

NCRT, neoadjuvant chemoradiotherapy; ESCC, esophageal squamous cell carcinoma; EC, esophageal cancer; OS, overall survival; TNM, tumor node metastasis; BMI, body mass index; PNI, prognostic nutrition index; SII, systemic immune-inflammation index; WBC, white blood cell; RBC, red blood cell; HB, hemoglobin; PLT, platelet count; AMC, absolute monocyte count; ANC, absolute neutrophil count; ALC, absolute lymphocyte count; PLR, 
platelet lymphocyte ratio; NLR, neutrophil lymphocyte ratio; LMR, lymphocyte monocyte ratio; NRI, net reclassification improvement; IDI, integrated discrimination improvement.

\section{Ethics Statement}

Our study was approved by The Shantou University Medical Ethics Committee (approval no. SUMC2011XM-0066). All patients provided written informed consent prior to enrollment in the study.

\section{Funding}

This work was funded by grants from Science and Technology Planning Project of Shantou City (No. 200605115266724) and 2020 Li Ka Shing FoundationCross-Disciplinary Research Grant (No. 2020LKSFG01B).

\section{Disclosure}

The authors declare that they have no competing interest.

\section{References}

1. Enzinger PC, Mayer RJ. Esophageal cancer. $N$ Engl J Med. 2003;349 (23):2241-2252. doi:10.1056/NEJMra035010

2. Pennathur A, Gibson MK, Jobe BA, Luketich JD. Oesophageal carcinoma. Lancet. 2013;381(9864):400-412. doi:10.1016/S01406736(12)60643-6

3. Zhang HZ, Jin GF, Shen HB. Epidemiologic differences in esophageal cancer between Asian and Western populations. Chin J Cancer. 2012;31(6):281-286. doi:10.5732/cjc.011.10390

4. van Hagen P, Hulshof MC, van Lanschot JJ, et al. Preoperative chemoradiotherapy for esophageal or junctional cancer. $N$ Engl $J$ Med. 2012;366(22):2074-2084. doi:10.1056/NEJMoa1112088

5. Shapiro J, van Lanschot J, Hulshof M, et al. Neoadjuvant chemoradiotherapy plus surgery versus surgery alone for oesophageal or junctional cancer (CROSS): long-term results of a randomised controlled trial. Lancet Oncol. 2015;16(9):1090-1098. doi:10.1016/ S1470-2045(15)00040-6

6. Sjoquist KM, Burmeister BH, Smithers BM, et al. Survival after neoadjuvant chemotherapy or chemoradiotherapy for resectable oesophageal carcinoma: an updated meta-analysis. Lancet Oncol. 2011;12 (7):681-692. doi:10.1016/S1470-2045(11)70142-5

7. Morita M, Toh Y, Saeki H, et al. Clinical significance of chemoradiotherapy and surgical resection for cT4 esophageal cancer. Anticancer Res. 2012;32(8):3275-3282.

8. Liao Z, Zhang Z, Jin J, et al. Esophagectomy after concurrent chemoradiotherapy improves locoregional control in clinical stage II or III esophageal cancer patients. Int J Radiat Oncol Biol Phys. 2004;60 (5):1484-1493. doi:10.1016/j.ijrobp.2004.05.056

9. Nygaard K, Hagen S, Hansen HS, et al. Pre-operative radiotherapy prolongs survival in operable esophageal carcinoma: a randomized, multicenter study of pre-operative radiotherapy and chemotherapy. The second Scandinavian trial in esophageal cancer. World J Surg. 1992;16(6):1104-9; discussion 1110. doi:10.1007/BF02067069

10. Burmeister BH, Smithers BM, Gebski V, et al. Surgery alone versus chemoradiotherapy followed by surgery for resectable cancer of the oesophagus: a randomised controlled phase III trial. Lancet Oncol. 2005;6(9):659-668. doi:10.1016/S1470-2045(05)70288-6
11. Urba SG, Orringer MB, Turrisi A, et al. Randomized trial of preoperative chemoradiation versus surgery alone in patients with locoregional esophageal carcinoma. J Clin Oncol. 2001;19(2):305-313. doi:10.1200/JCO.2001.19.2.305

12. Tepper J, Krasna MJ, Niedzwiecki D, et al. Phase III trial of trimodality therapy with cisplatin, fluorouracil, radiotherapy, and surgery compared with surgery alone for esophageal cancer: CALGB 9781. J Clin Oncol. 2008;26(7):1086-1092. doi:10.1200/JCO.2007.12.9593

13. Greene FL, Sobin LH. The TNM system: our language for cancer care. J Surg Oncol. 2002;80(3):119-120. doi:10.1002/jso.10114

14. Li Y, Lu Z, Che Y, et al. Immune signature profiling identified predictive and prognostic factors for esophageal squamous cell carcinoma. Oncoimmunology. 2017;6(11):e1356147. doi:10.1080/ 2162402X.2017.1356147

15. Li W, Liu J, Zhao H. Identification of a nomogram based on long non-coding RNA to improve prognosis prediction of esophageal squamous cell carcinoma. Aging (Albany NY). 2020;12(2):15 12-1526. doi:10.18632/aging.102697

16. Yang Y, Huang X, Zhou L, et al. Clinical use of tumor biomarkers in prediction for prognosis and chemotherapeutic effect in esophageal squamous cell carcinoma. BMC Cancer. 2019;19(1):526. doi:10.11 86/s12885-019-5755-5

17. Zheng Y, Fu S, He T, et al. Predicting prognosis in resected esophageal squamous cell carcinoma using a clinical nomogram and recursive partitioning analysis. Eur J Surg Oncol. 2018;44(8):1199-1204. doi:10.1016/j.ejso.2018.04.011

18. Zhang H, Guo XW, Yin XX, Liu YC, Ji SJ. Nomogram-integrated C-reactive protein/albumin ratio predicts efficacy and prognosis in patients with thoracic esophageal squamous cell carcinoma receiving chemoradiotherapy. Cancer Manag Res. 2019;11:9459-9468. doi:10.2147/CMAR.S228113

19. Olingy CE, Dinh HQ, Hedrick CC. Monocyte heterogeneity and functions in cancer. $J$ Leukoc Biol. 2019;106(2):309-322. doi:10. 1002/JLB.4RI0818-311R

20. Gouveia-Fernandes S. Monocytes and macrophages in cancer: unsuspected roles. Adv Exp Med Biol. 2020;1219:161-185.

21. Kovacs AR, Pal L, Szucs S, et al. [Phagocytic function of monocytes and neutrophil granulocytes in ovarian cancer]. Orv Hetil. 2018;159 (33):1353-1359. Hungarian.

22. Blot E, Chen W, Vasse M, et al. Cooperation between monocytes and breast cancer cells promotes factors involved in cancer aggressiveness. Br J Cancer. 2003;88(8):1207-1212. doi:10.1038/sj.bjc.6600872

23. Coussens LM, Werb Z. Inflammation and cancer. Nature. 2002;420 (6917):860-867. doi:10.1038/nature01322

24. Shigeoka M, Urakawa N, Nakamura T, et al. Tumor associated macrophage expressing CD204 is associated with tumor aggressiveness of esophageal squamous cell carcinoma. Cancer Sci. 2013;104 (8):1112-1119. doi:10.1111/cas.12188

25. Bredenoord AJ, Fox M, Kahrilas PJ, et al. Chicago classification criteria of esophageal motility disorders defined in high resolution esophageal pressure topography. Neurogastroenterol Motil. 2012;24 (Suppl 1):57-65. doi:10.1111/j.1365-2982.2011.01834.x

26. Xiao FK, Wang L, Zhang WC, Wang LD, Zhao LS. Preoperative prognostic nutritional index is a significant predictor of survival in esophageal squamous cell carcinoma patients. Nutr Cancer. 2021;73 (2):215-220. doi:10.1080/01635581.2020.1757129

27. Takao K, Konishi H, Fujiwara H, et al. Clinical significance of prognostic nutritional index in the treatment of esophageal squamous cell carcinoma. In Vivo. 2020;34(6):3451-3457. doi:10.21873/invivo.12184

28. Liu X, Qiu H, Kong P, Zhou Z, Sun X. Gastric cancer, nutritional status, and outcome. Onco Targets Ther. 2017;10:2107-2114. doi:10.2147/OTT.S132432

29. Burgess NG, Wyeth JW. An audit of combined multichannel intraluminal impedance manometry in the assessment of dysphagia. J Gastroenterol Hepatol. 2011;26(Suppl 3):79-82. doi:10.1111/ j.1440-1746.2011.06655.x 
30. Alrawashdeh W, Jones R, Dumartin L, et al. Perineural invasion in pancreatic cancer: proteomic analysis and in vitro modelling. Mol Oncol. 2019;13(5):1075-1091. doi:10.1002/1878-0261.12463

31. Grivennikov SI, Greten FR, Karin M. Immunity, inflammation, and cancer. Cell. 2010;140(6):883-899. doi:10.1016/j.cell.2010. 01.025

32. Koike Y, Miki C, Okugawa Y, et al. Preoperative C-reactive protein as a prognostic and therapeutic marker for colorectal cancer. J Surg Oncol. 2008;98(7):540-544. doi:10.1002/jso.21154

33. Nakatani M, Migita K, Matsumoto S, et al. Prognostic significance of the prognostic nutritional index in esophageal cancer patients undergoing neoadjuvant chemotherapy. Dis Esophagus. 2017;30(8):1-7. doi:10.1093/dote/dox020

34. Candido J, Hagemann T. Cancer-related inflammation. J Clin Immunol. 2013;33(Suppl 1):S79-S84. doi:10.1007/s10875-012-98 47-0

35. Geng Y, Shao Y, Zhu D, et al. Systemic immune-inflammation index predicts prognosis of patients with esophageal squamous cell carcinoma: a propensity score-matched analysis. Sci Rep. 2016;6:39482. doi:10.1038/srep39482

36. Rice TW, Ishwaran H, Ferguson MK, Blackstone EH, Goldstraw P. Cancer of the esophagus and esophagogastric junction: an eighth edition staging primer. $J$ Thorac Oncol. 2017;12(1):36-42. doi:10.1016/j.jtho.2016.10.016

37. Hulscher JB, van Sandick JW, de Boer AG, et al. Extended transthoracic resection compared with limited transhiatal resection for adenocarcinoma of the esophagus. $N$ Engl J Med. 2002;347(21): 1662-1669. doi:10.1056/NEJMoa022343

38. Edge SB, Compton CC. The American Joint Committee on Cancer: the 7th edition of the AJCC cancer staging manual and the future of TNM. Ann Surg Oncol. 2010;17(6):1471-1474. doi:10.1245/s10434010-0985-4

39. Camp RL, Dolled-Filhart M, Rimm DL. X-tile: a new bio-informatics tool for biomarker assessment and outcome-based cut-point optimization. Clin Cancer Res. 2004;10(21):7252-7259. doi:10.11 58/1078-0432.CCR-04-0713

40. Vickers AJ, Cronin AM, Elkin EB, Gonen M. Extensions to decision curve analysis, a novel method for evaluating diagnostic tests, prediction models and molecular markers. BMC Med Inform Decis Mak. 2008;8:53. doi:10.1186/1472-6947-8-53

41. Pepe MS, Fan J, Feng Z, Gerds T, Hilden J. The Net Reclassification Index (NRI): a misleading measure of prediction improvement even with independent test data sets. Stat Biosci. 2015;7(2):282-295. doi:10.1007/s12561-014-9118-0

42. Hayashi K, Eguchi S. The power-integrated discriminant improvement: an accurate measure of the incremental predictive value of additional biomarkers. Stat Med. 2019;38(14):2589-2604. doi:10. 1002/sim. 8135

43. Ludmir EB, Palta M, Willett CG, Czito BG. Total neoadjuvant therapy for rectal cancer: an emerging option. Cancer Am Cancer Soc. 2017;123(9):1497-1506.

44. Cain H, Macpherson IR, Beresford M, et al. Neoadjuvant therapy in early breast cancer: treatment considerations and common debates in practice. Clin Oncol (R Coll Radiol). 2017;29(10):642-652. doi:10. 1016/j.clon.2017.06.003

45. Xu JZ, Wang WQ, Zhang SR, et al. Neoadjuvant therapy is essential for resectable pancreatic cancer. Curr Med Chem. 2019;26 (40):7196-7211. doi:10.2174/0929867325666180413101722

46. Yi Y, Li B, Sun H, et al. Predictors of sensitivity to chemoradiotherapy of esophageal squamous cell carcinoma. Tumour Biol. 2010;31 (4):333-340. doi:10.1007/s13277-010-0041-9

47. Tasdogan A, Faubert B, Ramesh V, et al. Metabolic heterogeneity confers differences in melanoma metastatic potential. Nature. 2020; 577(7788):115-120. doi:10.1038/s41586-019-1847-2
48. Chen PH, Cai L, Huffman K, et al. Metabolic diversity in human non-small cell lung cancer cells. Mol Cell. 2019;76(5):838-851.e5. doi:10.1016/j.molcel.2019.08.028

49. Muir A, Vander HM. The nutrient environment affects therapy. Science. 2018;360(6392):962-963. doi:10.1126/science.aar5986

50. Onodera T, Goseki N, Kosaki G. [Prognostic nutritional index in gastrointestinal surgery of malnourished cancer patients]. Nihon Geka Gakkai Zasshi. 1984;85(9):1001-1005. Japanese.

51. Nozoe T, Ninomiya M, Maeda T, et al. Prognostic nutritional index: a tool to predict the biological aggressiveness of gastric carcinoma. Surg Today. 2010;40(5):440-443. doi:10.1007/s00595-009-4065-y

52. Nozoe T, Kimura Y, Ishida M, et al. Correlation of pre-operative nutritional condition with post-operative complications in surgical treatment for oesophageal carcinoma. Eur J Surg Oncol. 2002;28 (4):396-400. doi:10.1053/ejso.2002.1257

53. Kanda M, Fujii T, Kodera Y, et al. Nutritional predictors of postoperative outcome in pancreatic cancer. Br J Surg. 2011;98 (2):268-274. doi:10.1002/bjs.7305

54. Yang Y, Gao P, Chen X, et al. Prognostic significance of preoperative prognostic nutritional index in colorectal cancer: results from a retrospective cohort study and a meta-analysis. Oncotarget. 2016;7(36):58543-58552. doi:10.18632/oncotarget.10148

55. Yang Y, Gao P, Song Y, et al. The prognostic nutritional index is a predictive indicator of prognosis and postoperative complications in gastric cancer: a meta-analysis. Eur J Surg Oncol. 2016;42(8):11 76-1182. doi:10.1016/j.ejso.2016.05.029

56. Chen $\mathrm{P}$, Wang $\mathrm{C}$, Cheng $\mathrm{B}$, et al. Plasma fibrinogen and serum albumin levels (FA score) act as a promising prognostic indicator in non-small cell lung cancer. Onco Targets Ther. 2017;10:3107-3118. doi:10.2147/OTT.S138854

57. Chen S, Yan H, Du J, et al. Prognostic significance of pre-resection albumin/fibrinogen ratio in patients with non-small cell lung cancer: a propensity score matching analysis. Clin Chim Acta. 2018; 482:203-208. doi:10.1016/j.cca.2018.04.012

58. Kos FT, Hocazade C, Kos M, et al. Assessment of prognostic value of "neutrophil to lymphocyte ratio" and "prognostic nutritional index" as a sytemic inflammatory marker in non-small cell lung cancer. Asian Pac J Cancer Prev. 2015;16(9):3997-4002. doi:10. 7314/APJCP.2015.16.9.3997

59. Mori S, Usami N, Fukumoto K, et al. The significance of the prognostic nutritional index in patients with completely resected non-small cell lung cancer. PLoS One. 2015;10(9):e0136897. doi:10. 1371/journal.pone.0136897

60. Elinav E, Nowarski R, Thaiss CA, et al. Inflammation-induced cancer: crosstalk between tumours, immune cells and microorganisms. Nat Rev Cancer. 2013;13(11):759-771. doi:10.1038/nrc3611

61. Talmor M, Mirza A, Turley S, et al. Generation or large numbers of immature and mature dendritic cells from rat bone marrow cultures. Eur J Immunol. 1998;28(3):811-817. doi:10.1002/(SICI)1521-4141(199803)28:03<811::AID-IMMU811>3.0.CO;2-S

62. Franklin RA, Liao W, Sarkar A, et al. The cellular and molecular origin of tumor-associated macrophages. Science. 2014;344 (6186):921-925. doi:10.1126/science.1252510

63. Kitamura T, Doughty-Shenton D, Cassetta L, et al. Monocytes differentiate to immune suppressive precursors of metastasis-associated macrophages in mouse models of metastatic breast cancer. Front Immunol. 2017;8:2004. doi:10.3389/fimmu.2017.02004

64. Qian BZ, Li J, Zhang H, et al. CCL2 recruits inflammatory monocytes to facilitate breast-tumour metastasis. Nature. 2011;475 (7355):222-225. doi:10.1038/nature10138

65. Shen SL, Fu SJ, Huang XQ, et al. Elevated preoperative peripheral blood monocyte count predicts poor prognosis for hepatocellular carcinoma after curative resection. BMC Cancer. 2014;14:744. doi:10.1186/1471-2407-14-744 
66. Huang SH, Waldron JN, Milosevic M, et al. Prognostic value of pretreatment circulating neutrophils, monocytes, and lymphocytes in oropharyngeal cancer stratified by human papillomavirus status. Cancer Am Cancer Soc. 2015;121(4):545-555.

67. Maeda R, Ishii G, Neri S, et al. Circulating CD14+CD204+ cells predict postoperative recurrence in non-small-cell lung cancer patients. J Thorac Oncol. 2014;9(2):179-188. doi:10.1097/JTO.00 00000000000044

68. Tsai YD, Wang CP, Chen CY, et al. Pretreatment circulating monocyte count associated with poor prognosis in patients with oral cavity cancer. Head Neck. 2014;36(7):947-953. doi:10.1002/hed.23400

69. Han LH, Jia YB, Song QX, et al. Prognostic significance of preoperative lymphocyte-monocyte ratio in patients with resectable esophageal squamous cell carcinoma. Asian Pac J Cancer Prev. 2015;16(6):2245-2250. doi:10.7314/APJCP.2015.16.6.2245

70. MacGuill M, Mulligan E, Ravi N, et al. Clinicopathologic factors predicting complete pathological response to neoadjuvant chemoradiotherapy in esophageal cancer. Dis Esophagus. 2006;19 (4):273-276. doi:10.1111/j.1442-2050.2006.00576.x

71. Rohatgi PR, Swisher SG, Correa AM, et al. Failure patterns correlate with the proportion of residual carcinoma after preoperative chemoradiotherapy for carcinoma of the esophagus. Cancer Am Cancer Soc. 2005;104(7):1349-1355.

72. Donahue JM, Nichols FC, Li Z, et al. Complete pathologic response after neoadjuvant chemoradiotherapy for esophageal cancer is associated with enhanced survival. Ann Thorac Surg. 2009;87 (2):392-399. doi:10.1016/j.athoracsur.2008.11.001

73. Dexter SP, Sue-Ling H, McMahon MJ, et al. Circumferential resection margin involvement: an independent predictor of survival following surgery for oesophageal cancer. Gut. 2001;48(5):667-670. doi:10.1136/gut.48.5.667
74. Okumura H, Uchikado Y, Matsumoto M, et al. Prognostic factors in esophageal squamous cell carcinoma patients treated with neoadjuvant chemoradiation therapy. Int J Clin Oncol. 2013;18(2):329-334. doi:10.1007/s10147-012-0383-y

75. Tong DK, Law S, Kwong DL, et al. Histological regression of squamous esophageal carcinoma assessed by percentage of residual viable cells after neoadjuvant chemoradiation is an important prognostic factor. Ann Surg Oncol. 2010;17(8):2184-2192. doi:10.1245/s10434-010-0995-2

76. Akutsu Y, Shuto K, Kono T, et al. The number of pathologic lymph nodes involved is still a significant prognostic factor even after neoadjuvant chemoradiotherapy in esophageal squamous cell carcinoma. J Surg Oncol. 2012;105(8):756-760. doi:10.1002/jso.23007

77. Qiu Q, Duan J, Deng H, et al. Development and validation of a radiomics nomogram model for predicting postoperative recurrence in patients with esophageal squamous cell cancer who achieved pCR after neoadjuvant chemoradiotherapy followed by surgery. Front Oncol. 2020;10:1398. doi:10.3389/fonc.2020.01398

78. Cai G, Yu J, Meng X. Predicting prognosis and adverse events by hematologic markers in patients with locally advanced esophageal squamous cell carcinoma treated with neoadjuvant chemoradiotherapy. Cancer Manag Res. 2020;12:8497-8507. doi:10.2147/CMAR.S257058

79. Shapiro J, van Klaveren D, Lagarde SM, et al. Prediction of survival in patients with oesophageal or junctional cancer receiving neoadjuvant chemoradiotherapy and surgery. $B r \quad J$ Surg. 2016;103 (8):1039-1047. doi:10.1002/bjs.10142

\section{Publish your work in this journal}

Cancer Management and Research is an international, peer-reviewed open access journal focusing on cancer research and the optimal use of preventative and integrated treatment interventions to achieve improved outcomes, enhanced survival and quality of life for the cancer patient.
The manuscript management system is completely online and includes a very quick and fair peer-review system, which is all easy to use. Visit http://www.dovepress.com/testimonials.php to read real quotes from published authors. 